\title{
Design of Timing Socket Based on Single Chip Microcomputer
}

\author{
HongBo Hao ${ }^{1}$, Shuailin Chen ${ }^{2}$ \\ ${ }^{1}$ Tianjin University of Science and Technology, China; \\ ${ }^{2}$ Qingdao University of Science and Technology, Shandong, China \\ E-mail:2844011096@qq.com \\ www.tust.edu.cn
}

\begin{abstract}
In this paper, the design of timing socket is mainly used for smart timing control of household appliances, and the working time of timing is set by pressing the key. In the working period, the external connection device and the power supply are in the conduction state. Outside the set time period, the external connector is disconnected from the power supply. This design uses STC89C52 as the driving chip. DS1302 clock module provides accurate timing. LCD1602 LCD module displays time and operation interface. The relay module controls the connection between the socket and the power supply. $D C$ power supply module is converted to DC power supply module.
\end{abstract}

Keywords: Smart timing control, STC89C52, DS1302 clock module, DC power, Socket

\section{Introduction}

Nowadays, with the improvement of living standards and the acceleration of life rhythm, the sockets on the market cannot meet our needs because of their single function. For example, electric vehicle charging is usually about 8 hours. If it is too long, it is easy to lose the battery. If it is too full, it is not enough to charge. If it is charged at night, you often don't want to go out from home to the garage to charge the car. In the daytime, they forget to turn on the power; the water tower at home forgets to pump water, which causes temporary inconvenience to life. The school's broadcast of getting up is delayed because the staff on duty overslept. The fish tank in the home has not been supplied with oxygen for too long, which causes the fish to die of lack of oxygen. Many fans or news enthusiasts miss the start-up time and miss the wonderful ball games, so it is not convenient to use it at night. Considering the above reasons, we urgently need a smart socket. This socket can supply power to electrical appliances regularly and cut off the power supply of electrical appliances outside working hours. In this way, the standby loss of electrical appliances can be solved, the purpose of saving electricity can be achieved, and the hidden danger of safety can be eliminated ${ }^{1}$.

At present, in the market, the technology of time controller is relatively advanced and complete. Time controller is widely used in all kinds of electrical appliances. In the field of smart socket, however, it is still in the stage of development. The smart socket is only limited to the knob timer, or the time control socket with larger volume designed by counter chip. This kind of socket timing time is single, the function is single, the timing accuracy is low and it is difficult to really meet the needs of our daily life. Now we need a powerful time control socket to meet the market demand and improve our life. With the development of modern electronic science and technology, low power consumption and other characteristics. The price of finished products has decreased, and has been widely used. Accurate display of real-time time and timing time, it has the memory function and the data will not be lost after power off. It not only achieves the function and reduces the cost, but also it let our daily life have a profound impact.

(C) The 2021 International Conference on Artificial Life and Robotics (ICAROB2021), January 21 to 24, 2021 


\section{Overall scheme design}

This chapter mainly discusses the overall scheme of the system and the design scheme of each module, including the control core, switch, display module, clock circuit and keyboard circuit. In this paper, STC89C52 is selected as the core component of this design. The basic timing control function is realized by using the flexible programming design and rich IO port of the single chip microcomputer and the accuracy of its control. The overall design of the system mainly realizes the following functions:

(1) Human computer interaction interface: through independent buttons to establish a complete set of human-computer interaction interface.

(2) Infrared remote control: the system can set two timing time through the infrared remote control, and It can be forced on / off to confirm the start timing function

(3) Buzzer prompt: when the power is on and off, there will be a buzzer prompt.

\subsection{Power supply scheme}

The design power supply is $+5 \mathrm{~V}$ DC power supply. In this paper, USB power cord is used for power supply, one end is connected with DC interface, the other end is connected to $+5 \mathrm{~V}$ power supply. The power supply mode can be computer USB, mobile phone charger, power bank, etc. The power supply is supplied by $220 \mathrm{VAC}$ to $5 \mathrm{VDC}$ regulated power supply.

\subsection{Relay circuit scheme}

Relay is a kind of electrical switch, which is actually a switch device with small current to control large current. It can not only protect the circuit, but also realize the function of safe conversion circuit. There are many kinds of relay, including electromagnetic relay, time relay, and temperature relay. This paper uses SRD-05VDC-SL-C relay $^{2}$.

\section{Hardware circuit design}

\subsection{Minimum system of single chip microcomputer}

The minimum system consists of reset circuit, clock circuit and STC89C2. Minimum system circuit as is shown in the Fig. 1 below. This system uses STC89C52 control chip. The single chip microcomputer not only has strong function and low power consumption, but also has strong anti-interference ability and high cost performance ratio ${ }^{3}$.

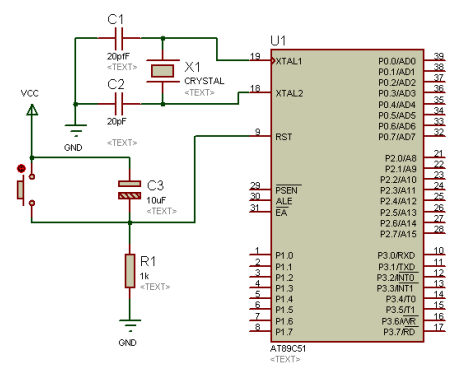

Fig.1. Minimum system circuit

\subsection{Clock circuit module}

The system needs to calculate the exact time, so the module uses an $11.0592 \mathrm{MHz}$ crystal oscillator. The clock circuit is used to generate the clock signal needed by MCU. In order to ensure the realization of synchronous working mode, the circuit should work in strict accordance with the time sequence under the control of the only clock signal. The clock circuit diagram is shown in Fig.2.

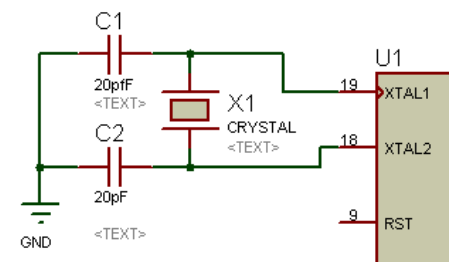

Fig.2. Clock circuit diagram

\subsection{Reset circuit}

Reset is the initialization operation of single-chip microcomputer. When the single-chip microcomputer starts and runs, it must be reset. The reset circuit includes two parts: power on reset and manual reset. The reset signal of MCU is high level reset. The reset circuit diagram is shown in Fig.3.

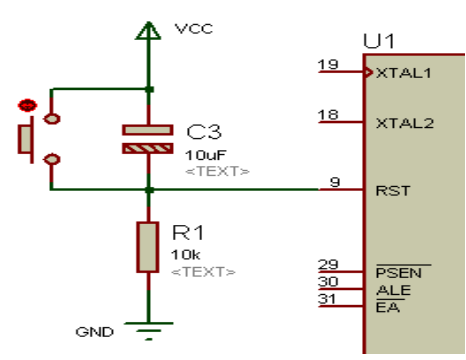

Fig.3. Reset circuit diagram 


\subsection{Socket power circuit}

The power on and off is not frequent, so this design uses the relatively low price relay as the control circuit device of socket power supply. An I / O port of the single chip microcomputer controls the on-off of the relay through a triode, so as to control the power on and off of the socket. The control circuit of socket power supply is shown in Fig.4.

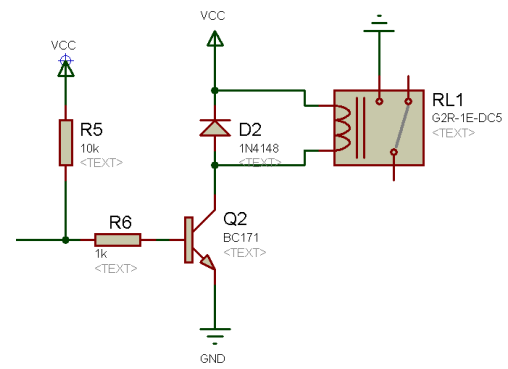

Fig.4. Socket power circuit

\subsection{Independent key module}

The key K1 is used to set the subtraction operation of the time value. The key $\mathrm{K} 2$ is used to set the addition operation of the time value. Key K3 can be set separately, and then select manual mode or automatic mode. The default mode of MCU is automatic mode. It can also work with the $\mathrm{K} 4$ button to set the position of the variable. Schematic diagram of key module is shown in Fig. $5^{4}$.

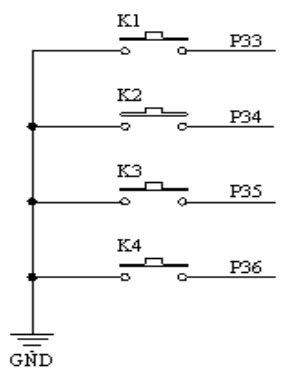

Fig.5. Schematic diagram of key module

\section{Software design}

\subsection{Main program flow chart}

In this design, the program is mainly under the control of single-chip microcomputer. In this process, the MCU first initializes, including setting the direction of each port, the initialization of each variable, the initialization of LCD, the disconnection of relay and the calibration of oscillation frequency.

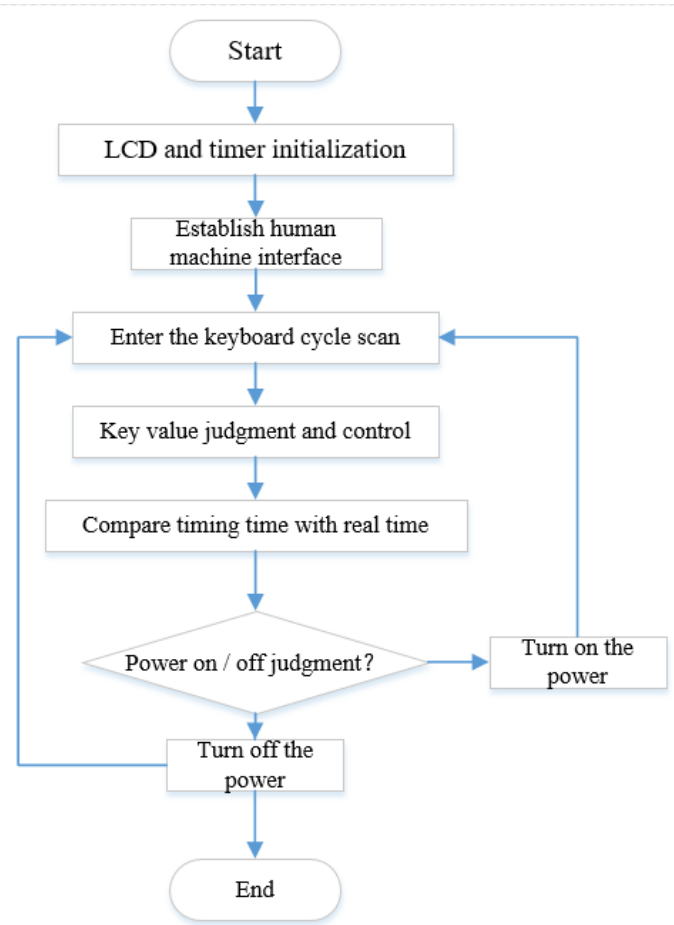

The flow chart of the whole system software design is shown in Fig.6.

Fig.6. Software design flow chart

\subsection{Design of setting time function}

The flow chart of setting timing is shown in Fig.7 below. After power on, enter the time timing program, and then judge whether the key is valid. If it is valid, set the start and end time of timing through four independent keys. If it is judged that the key is invalid, it will return to wait for the key program until the end of the setting. Set timing time program design: set function void key_with( ) ${ }^{5}$.

\section{Conclusion}

\subsection{System simulation and testing}

According to the designed hardware schematic diagram, the simulation circuit diagram is drawn in Proteus. Then the designed program is compiled and saved in. After running, the LCD can display the calendar time. The display page shows the year, month, day, 2019-05-17 in the down line, 15:30:51 in the uplink and w: 5 in the week. System simulation circuit diagram is shown in Fig.8 below ${ }^{6}$. 


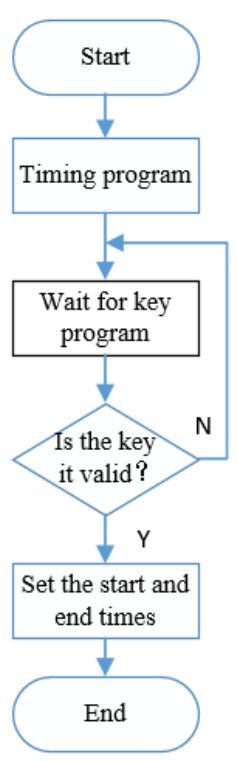

Fig.7. Schematic diagram of key module

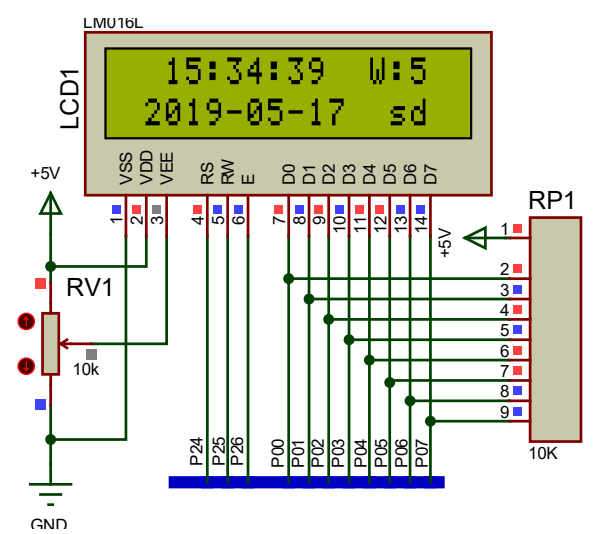

Fig.8. System simulation circuit diagram

\subsection{Power simulation and testing}

Multisim 13.0 is selected for the simulation and testing of DC voltage stabilizing circuit, which has rich simulation and analysis ability. This design first according to the hardware design circuit diagram, draws the circuit diagram in the Simulation Software Multisim, and then it carries on the operation simulation to the circuit. The waveform after running the simulation is shown in Fig.9.

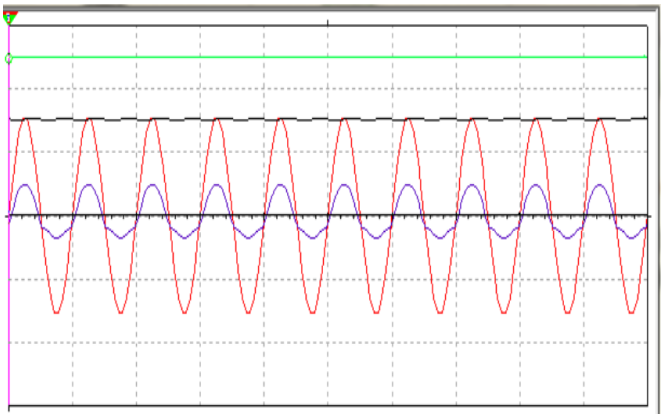

Fig.9. System simulation waveform

\section{References}

1. K. Tsai, F. Leu, I. You, Residence Energy Control System Based on Wireless Smart Socket and IoT, IEEE Access, vol. 4, pp. 2885-2894.

2. C. E. Shannon, A symbolic analysis of relay and switching circuits, Electrical Engineering, 57(12): pp. 713-723.

3. M. Wu, X. Wu, Design of the single-chip microcomputer experiment teaching system for application, 2011 International Conference on Electrical and Control Engineering, Yichang, 2011, pp. 649-652.

4. Kong, Fan Rong, Wang, Wenxin, Zhu, Jiajun. A Smart Home Control System Based on Controller Area Network Bus. 2013, 11(9):1667-1671(5).

5. Sandy Suryo Prayogo, Farid Al Rafi, Yulisdin Mukhlis. Design and Built IoT Home Panic Button for Smart City. 2019, 1175(1).

6. Hamid Mahmoudi, Mohsen Aleenejad, Reza Ahmadi. Reconfigurable rapid prototyping platform for power electronic circuits and systems for research and educational purposes. 2018, 11(7): 1314-1320. 